\title{
Filosofía pública y educación. La reconstrucción de la educación cívica en la democracia fuerte
}

FERNANDO BARCENA ORBE

Universidad Complutense de Madrid

\begin{abstract}
SUMMARY.- In this paper, the author explore the relevance and significance of civic education in a «strong democracy», in the context of european community. After explore and defend the moral condition of the Europe union, the author reflect on some specifics pedagogical commitments and responsibilities in this civic matter. Talk about a strong civic education is regarded as a opportunity to conform our moral character in the public's virtues and to increase the capacity of political and civic judgment. The pervivence of democracy in a technological age, as a social way of life, require the consolidation of a new public philosophy of education.
\end{abstract}

\section{INTRODUCCIÓN}

En los últimos años venimos asistiendo a un interesante debate dentro de la fílosofía moral y política, principalmente centrado en las nociones de lo público y lo privado. Es sabido que la dicotomía público-privado ha estado presente en casi toda la historia de la civilización occidental, porque para ser exactos debemos señalar que la distinción entre ambas esferas era desconocida en la polis. En efecto, para los griegos «hombre» $\mathrm{y}$ «ciudadano» significaban prácticamente lo mismo: «participar en la vida de la polis, de su ciudad, significaba 'vivir'» (G. Sartori, 1.987, pp. 352353).

Las discusiones y controversias que este asunto ha generado se han proyectado en la educación, a distintos niveles; pero dentro del estricto marco de la filosofía moral y política el debate en cuestión ha sido fundamentalmente protagonizado por dos frentes teóricos bien definidos. Por una parte, el de los teóricos del liberalismo, como Ronald Dworkin, para quien «el gobierno debe permanecer neutral ante lo que podemos llamar el problema de la vida buena» (R. Dworkin, 1977, p. 127); o como John Rawls, que claramente apuesta por la libertad como principio prioritario de lo que ha denominado «justicia como equidad» (J. Rawls, 1986).

El segundo enfoque está compuesto por los teóricos del comunitarismo, como Michael Sandel (1982) o Alasdair MacIntyre (1987 y 1990 b), entre otros, siendo el caso de este último especial, pues sin dejar de interesarse por aquellas formas polí- 
ticas mediante las cuales se puede personificar socialmente la vida virtuosa, en ningún momento su aspiración es — como en general la de los comunitaristas contemporáneos- ofrecer soluciones para la sociedad en su conjunto.

Recientemente, y de forma explícita, ha pedido no se confundan sus planteamientos con las tesis del moderno comunitarismo, aunque reconozca que su atención está puesta también, como la de aquéllos, en la Gemeinschaft (comunidad, grupo básico). En cualquier caso, le parece claro a MacIntyre que la actividad política debe hoy consistir «en la construcción y promoción en el ámbito local de formas de comunidad y de relaciones sociales basadas en la actividad práctica, en las cuales - y a través de las cuales- se consiguen los bienes inmanentes a la praxis» (A. Macintyre, 1990 a, p. 93).

Dentro del amplio espectro de temas discutidos entre liberales y comunitaristas, frecuentemente se ha invocado el nombre de Hannah Arendt, y especialmente por los defensores del comunitarismo. Arendt había expresado su opinión de que la esfera pública, mucho más que cualquier otro ámbito, constituía lugar propicio para el autodesarrollo: «ni la educación, ni la ingeniosidad, ni el talento pueden reemplazar a los elementos constitutivos de la esfera pública, que la hacen lugar propicio para la excelencia humana» (H. Arendt, 1974, p. 73). De acuerdo con este planteamiento general, existen algunos aspectos de su pensamiento ético-político que justificarían su inclusión en las filas del comunitarismo; como por ejemplo el hecho de que rechazase las tesis de la democracia representativa —en favor de una democracia participativa más directa — o elogiase la «tradición revolucionaria» defendiendo la necesidad de un mayor compromiso cívico del individuo y mayor atención a la deliberación y a la formación del juicio político, por su destacado papel en la conformación de un adecuado compromiso con una forma pública de ver el mundo (ver, H. Arendt, 1988, pp. 222 y ss.): «la capacidad de juzgar —decía— es específicamente una habilidad política»; «El juicio - hace notar - puede ser una de las habilidades fundamentales del hombre como ser político, en la medida que le habilite para orientarse en la esfera pública» (H. Arendt, 1984, p. 221)1. Ahora bien, similarmente a lo que acontece con MacIntyre, un análisis en profundidad del pensamiento político de Hannah Arendt permite afirmar, como señala d'Entreves (1989), que ni liberales ni comunitaristas pueden legítimamente incluir dentro de sus filas a esta autora. Para ella, la política —que es acción, y no trabajo o labor- no es un medio para la satisfacción de las preferencias individuales ni una forma de integrar a los individuos en una concepción simple o trascendental del bien. Su noción de la política se fundamenta en la idea de la ciudadanía activa; esto es, en el reconocimiento del valor e importancia del compromiso cívico y de la deliberación colectiva sobre los asuntos que afectan a la comunidad política (J. M. d'Entreves, 1989, p. 1). Concebida como una forma de acción, la política es la actividad relativa a la forma de llevar los asuntos de una comunidad por medio del lenguaje. La comunidad política, por tanto, constituye un grupo de personas unidas por el compromiso de llevar a cabo una determinada forma de vida política: aquélla que presupone una participación activa y creativa de los ciudadanos en la gestión de los asuntos comunes. Propiamente, esta

1. Sobre el juicio político y cívico, consúltese: S. BenHABIB, 1988, pp. 47-48; B. BARBER, 1988, pp. 196 y ss. y R. BEINER, 1983, pp. 12-19. 
comunidad política no equivale de hecho a la participación. La forma de vida política no supone participación per se, «sino aquél tipo de participación que emana de un compromiso con la forma pública de estar en el mundo y con todo lo que ello entraña» (B. Parekh, 1986, p. 26).

De acuerdo con estas ideas es más razonable asignar a nuestra autora a la tradición clásica del pensamiento cívico y originado en Aristóteles y modernamente afianzado en el republicanismo de Jefferson y los planteamientos de Tocqueville. De acuerdo con esta tradición, la política encuentra su auténtica expresión cuando los ciudadanos se reunen en un espacio público para deliberar, juzgar y decidir sobre los asuntos colectivos que les conciernen. En esta línea de pensamiento, la actividad política es valorada no tanto por su capacidad para llegar a acuerdos o descubrir una concepción global de lo bueno, sino en la medida que habilita a los ciudadanos para ejercer y desarrollar su capacidad de juicio cívico y político.

Recientemente estos planteamientos han encontrado un nuevo dinamismo en lo que Benjamín Barber (1984) ha denominado «democracia fuerte». En esencia, aquí se subrayará la centralidad de la dimensión de la democracia como forma de vida y la importancia de la educación cívica - cuyo núcleo será la formación en capacidad de juicio político- como fundamento de la democracia misma. Algunos estudios — como el de Richard Pratte (1988), por ejemplo- demuestran que estas tesis ya se han dejado sentir en la literatura pedagógica más relevante sobre la materia.

Teniendo en cuenta estas consideraciones generales, mi propósito en este artículo será explorar el sentido que podría y debería adoptar la práctica de la educación cívica - concebida como una ocasión para la intervención pedagógica - ante la tentativa de consolidar una «ética» acorde con la situación global que caracteriza a nuestra época - la progresiva tecnologización de las sociedades- y con las concretas circunstancias que rodean a nuestras aspiraciones democráticas en Europa. Dentro de este propósito general, ocupará un lugar central la cuestión de si es o no pertinente rechazar distinciones características de la sociedad y el liberalismo modernos como las de vida privada y pública. A esta pregunta se responderá afirmativamente, ya que lo contrario impediría superar uno de los principales defectos del liberalismo, a saber: su incapacidad para proporcionar pautas morales suficientemente fundadas y para reconocer que en todas las áreas de la vida y la actividad humana son necesarias las mismas virtudes. El interés de la ética es el ser humano en cuanto tal, la persona en su integridad, y no sólo como individuo que desempeña un rol social determinado.

Plantearé además la cuestión de la educación cívica en estrecha conexión con el problema de la formación de carácter moral - y todo ello dentro de lo más valioso que el discurso comunitarista puede contener - lo cual parece sumamente coherente con el hecho de que el planteamiento de la cuestión de la unidad europea usualmente se hace refiriéndola a la idea de la comunidad, lo que sin más es un claro síntoma de que en la actualidad vivimos —o padecemos, según se mire- una verdadera inflación del discurso comunitarista. 


\section{LA CONSTRUCCIÓN DE EUROPA COMO OBJETIVO MORAL}

\section{La búsqueda de lo común en la construcción de una ciencia del ser humano}

No se añade dato nuevo alguno a lo que quizá ya sepamos si se afirma que la tarea de la construcción de una Europa unida no constituye sólo un problema económico, monetario o financiero. Es indiscutible que referirse a ella exige dar cuenta de diversos niveles y ámbitos de «unidad». Entre ellos, hay que añadir al ya mencionado, principalmente, el político, la unidad social y también la cultural. Pero construir una Europa unida es, prioritariamente y ante todo, una labor que afecta muy directamente a nuestras vidas como ciudadanos de Europa. En ella estamos implicados todos, porque a todos afectará pensar Europa en clave de unidad y comunidad. Se une lo diferente y lo diverso, y como ha sabido ver Gadamer (1990) este hecho impone sus condiciones, pues «también en los otros y los de otra clase - dice- se puede establecer una especie de encuentro con uno mismo. Más apremiante que nunca es, sin embargo, la tarea de aprender a reconocer lo común en los otros y los de otra clase» (p. 116).

Esto que es «común» sin embargo no puede quedar reducido a un mero «sistema racional de utilidades» o una especie de «religión de la economía mundial», como agudamente hace notar el filósofo alemán. Al menos debería contribuir a la construcción de una verdadera ciencia del ser humano que exija de nosotros, como ciudadanos de Europa éticamente comprometidos, virtud política y humana capaz de regular nuestras relaciones en el marco de un cada vez más fuerte interculturalismo: «La ciencia del ser humano en toda su diversidad se convertirá - matiza Gadamer- en una tarea moral y filosófica para todos nosotros» (p. 116). Por ello mismo ha de consistir también, y especialmente para los educadores, en un objetivo moral cuya consecución mueva a la revisión crítica de una buena parte del pensamiento pedagógico y la puesta en práctica de diversas iniciativas educativas. Entre ellas se sitúa en lugar privilegiado la dimensión de la educación o formación del núcleo social y político del hombre, uno de cuyos aspectos centrales, sin duda, lo constituye la formación cívica.

\section{El tratamiento actual de la educación cívica. Determinación de obstáculos}

Ahora bien, hablar de la educación cívica es en sí mismo una verdadera fuente de polémica y controversia. La variedad de enfoques teóricos y de investigación actualmente disponibles sobre la materia así parece corroborarlo, haciendo de la necesidad de adoptar un enfoque teórico de investigación sobre la materia una difícil decisión (National task force on citizenship education, 1977, pp. 180-184). Por otra parte, la tentativa de reformular la noción de ciudadanía - y por tanto el sentido mismo de la educación cívica - teniendo como marco teórico inspirador la concepción de la democracia como forma de vida, choca además con otra serie de dificultades. Destacaremos aquí las siguientes. 


\section{a) Obstáculos sociales}

Para Pratte (1988), cualquier intento por revitalizar una cultura cívica inspirada en la idea de la democracia como forma de vida - tal y como ésta pudo haber sido formulada en el pensamiento aristotélico y continuada en la tradición de pensamiento que representó la filosofía pública del republicanismo americano- choca con un importante obstáculo social, que deriva del tipo de cultura democrática hoy dominante en las estructuras sociales y las instituciones contemporáneas. Estas parecen favorecer una forma de vida pública y política propiciatoria de la separación entre el trabajo y la satisfacción moral de realizarlo, como contribución a la formación personal del individuo; entre los aspectos intelectuales y los emocionales de la formación, entre la acción individual y el gusto por la acción social y colectiva. En suma, una estructura de vida social fragmentada, donde la aspiración al pluralismo reafirma aún más nuestra separación y la atomización moral de la comunidad. Como consecuencia de tal fragmentación de la vida social, se impide en primer término la consecución de un ethos social mínimamente unificado capaz de vincular lo diverso y de abrir espacios para la búsqueda de lo común en experiencias significativas de aprendizaje informal. La carencia de estos ethos, complica enormemente la tarea de explicar en lo que deba consistir una sociedad justa, a parte de que vuelve más espinosa aún la pretensión de reproducir en nuestros días una democracia directa al estilo griego. En segundo lugar, la fragmentación de la vida social se encuentra cada vez más presidida de una cultura individualista de la competencia, que lejos de consistir en una competencia animada por la búsqueda de la excelencia moral, consiste en una competitividad en la que siempre hay ganadores y perdedores. Nuestra sociedad ha perdido de vista la existencia de los bienes compartibles y la necesidad de ejercer las distintas prácticas sociales como actividades dotadas de bienes inherentes compartibles, cuya consecución consiguen el sano efecto de lograr un bien para todos.

\section{b) Obstáculos filosóficos}

En estrecha relación con lo anterior, este tipo de obstáculos derivan de la preeminencia, en el plano teórico y filosófico, de una tradición democrática y social individualista dentro de la cual predomina una concepción de la vida social - también fragmentada - que reafirma la escisión entre la vida privada y la vida pública, la autoidentidad y la identidad colectiva, la noción de buena persona y la de buen ciudadano. Se trata de una concepción de la democracia que acentúa sólo el carácter protector de ésta. Es una democracia débil en la que el ciudadano se limita a participar en la elección de sus representantes, pero en ningún caso a comprometerse activa y responsablemente en el diálogo público y en la deliberación compartida sobre asuntos de interés común. Todo lo más, el ciudadano es capaz de elaborar juicios privados sobre asuntos públicos. El compromiso cívico es individual, pero sin trascender al exterior, como contribución personal y solidaria a la mejora de la calidad de la vida y las prácticas sociales. 


\section{c) Obstáculos institucionales de la escuela}

Esta tercera categoría de obstáculos están relacionados con un modo estrecho de concebir las responsabilidades educativas de la escuela, cuando sobre ésta pesa una visión estrictamente gerencial según la cual - a través de una enseñanza defensiva- se hace de la «educación» una mera evitación en el alumno de comportamientos disociales y una ocasión para compartamentalizar los aprendizajes según categorías y criterios que no responden precisamente a la idea de una práctica educativa unitaria y contínua, sino dispersa y discontinua. La cuestión de la implantación de una nueva idea de ciudadanía a través de una sólida educación cívica se complica aún más debido a la ausencia de criterios claros que permitan evaluar con garantías el significado y eficacia de la concepción de la civilidad en sentido fuerte y participativo. Tal vez por ello resulta explicable la compleja y asimismo diversa situación en la que se encuentra la enseñanza de la moralidad y del civismo, especialmente en el ámbito escolar europeo. Dedicaremos a ello unas breves palabras.

En efecto, puede decirse que son tres los rasgos que caracterizan este asunto en los sistemas educativos europeos. En primer lugar, el débil estatus institucional de la educación moral y cívica en el conjunto de los países de Europa y la pluralidad real de relaciones asignadas, desde el punto de vista curricular y disciplinar, a la religión, la moral y la civilidad. En segundo término, la preeminencia de una orientación formalista - no centrada en el contenido - en los programas de educación moral, prevalencia ésta que, sobre todo, hace que prime el objetivo del desarrollo del razonamiento y del juicio moral, tendiendo a incluir en ese ámbito formativo, por lo demás, aspectos estrictamente vinculados a la formación del ámbito cívicopolítico. Finalmente, la escasa claridad $\longrightarrow 0$ en todo caso fuerte polémica - sobre dos cuestiones paralelas: primero, si la disciplina encargada de la enseñanza de estos aspectos debería concretarse como una materia específica o debería encontrarse implícitamente esparcida a lo largo de todo el currículo (como es el caso de la opción española en la Reforma); y, segundo, si tal disciplina debería tener una orientación normativa o una base común que asegure en todo caso la existencia de un consenso mínimo o mínimo moral, que se concretaría en la enseñanza relacionada con los Derechos Humanos (A. Bolívar Botia, 1990, pp. 8-13).

Sea como fuere, no podemos entrar aquí en la discusión detenida y pormenorizada de este conjunto de problemas, que tan sólo quedan apuntados para ilustrar las dificultades propias del tema. No obstante, hay que subrayar una idea central. La unión de lo diverso, o la búsqueda de lo «común» en un espacio marcado por un fuerte interculturalismo, debe pasar por la acentuación de una estrecha relación entre la educación cívica y la enseñanza de los Derechos Humanos, al objeto de evitar que predomine un espíritu excesivamente particularista, que por naturaleza es contrario a la consecución de la unidad europea. De hecho, tal y como se hacía notar en la Convención Europea para la salvaguarda de los Derechos del Hombre y de las Libertades Fundamentales (1950), sabemos que en el seno del Consejo de Europa se reconocía como medio fundamental para la realización y fortalecimiento de la unidad de sus miembros signatarios «la salvaguarda y el desarrollo de los Derechos del Hombre y de las libertades fundamentales». De este modo, es exigible que la compatibilidad con los Derechos Humanos llegue a convertirse en un firme criterio 
de definición de las metas o fines generales de la educación, pues ninguna finalidad educativa nacional podría considerarse moralmente legítima si estuviese en desacuerdo con la Declaración Universal de Derechos Humanos. Por eso, como recuerda Wheeler (1976) «es posible, y necesario, enseñar que la lealtad al propio país es compatible con la preocupación por el mundo entero, y que los intereses nacionales sufrirán inevitablemente si se ignoran los intereses internacionales» (p. 95).

\section{HACIA UNA FILOSOFÍA PÚBLICA}

\section{Civilidad y ethos democrático}

Cualquiera que sea la vocación particular de una persona, o la profesión que desarrolle, es decir, cualquiera que sea la esfera en la que un individuo es activo, «todos los miembros de un cuerpo político democrático pueden también relacionarse a nivel práctico con la esfera política», hace notar Agnes Heller (1989, p. 218). Esta circunstancia exige extender el razonamiento moral a las razones impersonales, o para decirlo con mayor exactitud: a las razones que son de todos, y no sólo de una individualidad personal, porque el razonamiento moral también consiste en la capacidad de mirar y percibir con ojos objetivos todo lo más sujetivo que existe en el mundo. De esta suerte, como ciudadanos tenemos la posibilidad de «juzgar la política»: la juzgamos en la medida que nos concierne. Ello supone relacionar la competencia cívica con la configuración de una ética pública y ciudadana a través de la cual se forme en la capacidad de juicio al individuo. Ahora bien, para que el hombre pueda con cierto éxito actuar políticamente como tal ciudadano, se precisa además la práctica de ciertos hábitos sociales, virtudes cívicas o públicas, como las ha llamado recientemente Victoria Camps (1990). Así, incluso admitiendo que la democracia es infundada e infundable (en el sentido que consiste en una «creación y elección gratuita» que ningún principio utilitarista conseguirá demostrar); e incluso reconociendo la intrínseca fragilidad de la democracia, igualmente tendremos que admitir que su posible y tal vez única «garantía» reside en el ethos democrático extendido y generalizado. Sin las virtudes del ciudadano, la actualidad y vitalidad de la democracia será permanentemente puesta en peligro. Más aún, «el ethos democrático, de hecho, se produce y refuerza, sólo a través del ejercicio práctico de los derechos democráticos. A través de la participación activa. A través de una activa pasión por la democracia» (Flores D’Arcais, 1990, p. 13).

Así pues, hay una estrecha conexión entre la conformación de un sólido ethos democrático y la encarnación de la democracia misma en vida social, más allá de comprensión como una pura forma de gobierno o forma política. Para Adela Cortina (1990), este punto es claro: «sólo un ethos solidario, una actitud solidaria ante la vida social, puede alumbrar una democracia sin frustraciones» (p. 257). Este «ethos solidario» es un componente nuclear de la ética democrática, una ética capaz de asegurar una participación real, y no ficticia, de todos los ciudadanos en la esfera pública, bien a través de su acción directa o de auténticos representantes de sus intereses. Ahora bien, de acuerdo con lo que dijimos al comienzo - sobre la imposibilidad real de reproducir en nuestro tiempo una democracia participativa como la 
de la polis griega - hay que realizar algunas matizaciones encaminadas a plantear dicho ethos democrático y tal participación en la esfera pública de un modo realista. En este sentido, me parece que el planteamiento esencial de MacIntyre puede sernos en extremo útil aquí.

En efecto, de lo que se trataría es de construir y promover en el ámbito local formas de comunidad basadas en prácticas, esto es, en actividades o formas de praxis dotadas de y perseguidas por sus bienes inmanentes e internos. La práctica del diálogo público, por ejemplo, no sería una actividad encaminada a lograr un consenso estratégico dentro del cual primasen ocultos intereses personales, sino una actividad en la que realmente buscamos ver satisfechos los intereses de todos y enriquecido el bien público. La creación y conservación de este tipo de relaciones comunitarias - como el lugar de trabajo, la familia, la vecindad, etc.— resultan así actividades con sus propios bienes internos. «Cada una de ellas —observa MacIntyre- tiene sus propias relaciones con otras estructuras semejantes, su propia estructura de autoridad, su modo genuino de educar a sus miembros a obedecer racionalmente a la autoridad y a ejercerla de modo inteligente» (A. MacIntyre, $1990 \mathrm{a}$, p. 92). Pues bien, aunque ocasionalmente la defensa y mantenimiento de estas formas locales de comunidad exija algún tipo de participación ad hoc en los sistemas políticos globales, lo importante es que la participación queda limitada —concretada — a situaciones muy precisas; y esto es tanto como defender una participación real y directa.

\section{La pervivencia de la democracia en una era tecnológica}

Este ethos, por otro lado, es actualmente una clara necesidad. En efecto, vivimos hoy en una sociedad altamente tecnificada e incluso aspiramos poder iniciar un nuevo milenio jactándonos de unas capacidades tecnológicas que superen lo hasta ahora imaginado. Pero estos logros tecnológicos plantearán, de hecho, nuevos y problemáticos retos éticos, de difícil solución en muchos casos. La pregunta, entonces, parece inevitable: ¿Cómo tratar en una sociedad democrática, en la que los ciudadanos deben decidir la dirección moral que ha de tomar la sociedad, los nuevos desafíos éticos que una época altamente tecnificada planteará?; ¿Cómo pueden los ciudadanos sostener y sustentar el control de la política si, quizá como es previsible, cada vez más se verán gobernados por «expertos»?

Como hace notas John W. Cooper (1989), una correcta respuesta a estos interrogantes debe pasar por admitir y asimilar claramente la realidad del cambio. Por eso la respuesta al interrogante sobre las posibilidades de la supervivencia de la democracia en una era altamente tecnificada se debe obtener a través de la obtención de un juicio claro - en el que todos estamos comprometidos- sobre cuestiones tales como el papel crítico que la ética profesional está llamada a jugar en la formación inicial y permanente de las personas; la fuerza creativa entre los forjadores de opinión pública en áreas como la política, la economía, la cultura, etc.; y la naturaleza misma de la sociedad democrática pluralista (pp. 47-48).

Es justamente dentro de esta última cuestión donde en la tarea de la educación cívica juega un papel esencial la formación del juicio cívico-político - como entre otros han sabido mostrar Oakeshott (1962), Beiner (1983) y Barber (1984 y 1988) - y a partir del cual cabe insistir en la necesidad de que dicha 
educación llegue a formar parte realmente significativa de una nueva filosofía pública que reconozca la intrínseca vitalidad y dinamismo de la democracia como forma de vida social.

Como consecuencia de ello, hay que hablar de la civilidad —del civismo y de la educación cívica - como de un particular imperativo, asunto del que me ocuparé en el siguiente apartado.

\section{EL IMPERATIVO CÍVICO}

\section{El sentido de una nueva cultura cívica}

La literatura especializada sobre la cuestión que estamos tratando es realmente fructífera y abundante. Principalmente en los Estados Unidos, aunque también en Italia, Francia e Inglaterra, viene hablándose en los últimos años de lo que Richard Pratte (1988) ha llamado «the civic imperative», que básicamente pide la construcción de una nueva cultura cívica consonante con las condiciones objetivas bajo las cuales vivimos, así como con nuestros ideales morales superiores. La construcción de tal cultura cívica - parte de lo que Sullivan (1982) ha incluido en su petición de «reconstruir la filosofía pública»— no es, sin embargo, una cuestión puramente técnica. Su creación no sólo reclama una superficial o formal educación cívica —un conocimiento del funcionamiento y organización de las instituciones y el ejercicio fáctico del derecho al voto - sino toda una transformación del carácter que habilite al ciudadano para juzgar las realidades donde vive y, como he dicho, evaluar y juzgar la política desde un particular talante moral.

En este punto, la construcción de una nueva cultura cívica exige transformar el carácter mediante el ejercicio habitual de virtudes públicas —como la tolerancia, la solidaridad, la responsabilidad solidaria, la valentía cívica, la justicia, la disposición a la comunicación racional y la phrónesis (saber práctico) - y adentrarse en la aventura de configurar una ética pública y ciudadana coherente con el hecho - que particularmente se me antoja crucial - de que la democracia es, sobre todo, una «forma de vida», y no sólo una forma de gobierno: «Una democracia —escribió Dewey (1982) - es más que una forma de gobierno; es primariamente un modo de vivir asociado, de experiencia comunicada juntamente. La extensión en el espacio del número de individuos que participan en un interés, de modo que cada uno ha de referir su propia acción a la de los demás y considerar la acción de los demás para dar pauta y dirección para la propia, equivale a la supresión de aquellas barreras de clase, raza y territorio nacional que impiden que el hombre perciba la plena significación de su actividad» (p. 98) ${ }^{2}$. Concebir la democracia desde este ángulo tiene fuertes implicaciones teóricas y prácticas para la institucionalización de la educación cívica. Pues es claro que una vez lograda una mayor individualización y ampliación de la comunidad de intereses, la tarea de sostener y extender esta forma de vida constituye, antes que nada, una actividad esforzada, consciente y deliberada

2. Ver también, J. Dewey, 1954, p. 148 y ss; Ch. BeRRY, 1989, pp. 38-65 y 94-114 y C. G. RyN, 1990, pp. 3-26 y 81-90. 
encaminada, por ejemplo, a hacer que todos los ciudadanos tengan iguales oportunidades intelectuales y que sean educados para la iniciativa y la participación crítica y creativa en el ámbito público y social. Concebir la democracia como «forma de vida» exige, de esta suerte, la consolidación de una educación cívica que habilite al ciudadano para una participación social creativa y dinámica.

Como ya se mencionó, algunos estudios americanos se han referido a este tema defendiendo la tesis de la democracia fuerte, expresión con la cual viene a distinguirse una forma de democracia participativa conectada con la idea del autogobierno comunitario de los ciudadanos, los cuales se sienten unidos menos por un conjunto de intereses homogéneos que por una sólida educación cívica que les habilita para la consecución de propósitos que comparten a través de actitudes cívicas e instituciones participativas (B. Barber, 1984, p. 117 y ss.).

\section{La sensibilidad de Europa hacia la ciudadanía participativa}

La centralidad de esta dimensión de la educación - la educación de lo que para nuestro caso tal vez podría denominarse «cuidadanía europea»- también viene siendo destacada en variedad de Informes Europeos. Así lo han hecho, por ejemplo, los grupos Socialistas y Popular del Parlamento Europeo en diversos momentos y con motivo de la presentación de distintos Programas y Manifiestos (Grupo Socialista Europeo, 1989, p. 19 y Partido Popular Europeo, 1989, p. 46).

Por otra parte, en la Cuarta Conferencia de Ministros de Educación de los Estados Miembros de la Región Europea (1988), celebrada en París en septiembre de 1988, se señalaba que el debate público sobre la educación ponía de relieve algunas novedades de importancia, destacándose «el cometido mucho mayor que se asigna a la educación en las actividades destinadas a resolver ciertos problemas sociales; ello se hace cada vez más evidente, por ejemplo, en el mayor interés por la educación ética, moral y cívica y en la inclusión en los programas de aspectos nuevos de educación preventiva en materia de seguridad, medio ambiente, uso indebido de drogas, salud, etc.» (p. 5). Y así, se recomendó a los Estados Miembros que «refuercen el carácter humanista de la educación en su dimensión internacional mediante el estudio detenido de los problemas mundiales contemporáneos, de los importantísimos retos que plantean a la humanidad — salvaguardia de los derechos de la persona humana, defensa de la paz, preservación de medio ambiente, fomento del desarrollo- y de las posibilidades de cooperación internacional para afrontarlos» (p. 53). Paralelamente, el Informe anual de 1988 del Consejo de Cooperación Cultural del Consejo de Europa, destaca como su primer gran dominio de acción la promoción de la identidad europea, dentro de la cual se reconoce a la diversidad cultural como un componente esencial (Conseil de L'Europe, 1989, p. 14). Esta idea, se reitera y refuerza asimismo en la «Carta Magna de las Universidades Europeas», pues como recuerda Caputo (1988) «construir Europa significa ante todo formar ciudadanos europeos, es decir, personas que, reconociendo una vocación e identidad cultural comunes, puedan imponer a las autoridades de sus estados los sacrificios indispensables, las limitaciones de soberanía necesaria para la integración. Europa será ante todo Europa de los pueblos y de las culturas, o no será nada» (p. 76). 


\section{La naturaleza de la competencia cívica en la democracia fuerte}

Ahora bien, es innegable que no existe teoría alguna de la competencia cívica que no responda a lo que, en general, debe ser un ciudadano educado en el marco de una sociedad democrática, pluralista y, además, intercultural. Pero es muy distinto referirse a la educación cívica desde el supuesto que concibe a la democracia exclusivamente como una forma de gobierno, que formularla teniendo en cuenta que la democracia, además y sobre todo, es una forma de vida. En sentido fuerte, la democracia es una forma de vida. Y en sentido fuerte también la ciudadanía ha de concebir al individuo como sujeto maduro educado para incorporarse en un diálogo público y en una participación real en su comunidad. Plantear la democracia desde este segundo punto de vista tiene indudables ventajas, morales y de carácter pedagógico, para nuestro tema. Las principales podrían ser las siguientes:

a) En primer lugar, concebir la democracia como forma de vida exige el recurso a la acción del ciudadano. Esta acción es bien particular y característica, pues se trata sin duda de una verdadera «acción» —es decir, una praxis, en sentido griegoque debe llevar a su activa participación en la construcción cultural y moral de la sociedad y en el sostenimiento de la propia democracia. Todo ello exige educación. Una educación que sepa mostrar y transmitir significativamente a los individuos los conocimientos, actitudes y valores esenciales que les capaciten para actuar como ciudadanos competentes, ilustrados y moralmente formados (A. Gutman, 1987, pp. 71-94 y 282-292).

b) En segundo término, entendida como forma de vida, la democracia, que exige como hemos visto una sólida formación cívica de índole participativa, a su vez reclama superar una concepción meramente individualista de la vida democrática para ir hacia un comunitarismo - no colectivista - en el que las virtudes públicas de la tolerancia y la solidaridad nos sostengan en la búsqueda pertinente de lo bueno para toda la sociedad. Esto último también reclama de la educación, y concretamente de una educación moral que, como mínimo, permita asentar las bases de una ética pública comprometida en el bien de todos y en la construcción de una buena sociedad. No hay más que reparar en el fenómeno del inter o multiculturalismo para darse cuenta de la necesidad de motivar el ejercicio habitual de la tolerancia, la solidaridad, el servicio, etc., a través de una buena educación que incida llamativamente en esta dimensión «cívica» del hombre a la que nos referimos ${ }^{3}$. En este sentido, una de las claves determinantes de la supervivencia de las instituciones libres en una democracia se encuentra en la relación de la vida privada con la vida pública. Es decir, el modo en que aquélla prepara al individuo para que, como ciudadano, participe en esta última. Pero también exige la vinculación entre la participación local de los ciudadanos con el diálogo nacional. Ello exige un cambio en la noción misma de la «política», que incorporaría de este modo la idea de que el individuo también alcanza su plenitud, en el marco de las relaciones con los demás, dentro de una sociedad organizada por medio del diálogo público (R. Bellah, p. 289).

3. Sobre el carácter y naturaleza de las «comunidades éticas», puede consultarse: G. BELLO, 1990, pp. 11-76 y V. HERNÁNDEZ, 1990, pp. 77-110. 


\section{Etica ciudadana y educación del juicio cívico}

Llamar a la «participación» —dentro de la cual la capacidad de juicio debe ocupar un lugar preeminente - y a una ampliación del sentido de «comunidad» es central para la tarea de construcción de una Europa unida. Ambas ideas se encuentran en el centro de esa «nueva sensibilidad» a la que se ha referido A. Llano (1988) señalando que «la indudable emergencia del ethos profesional, con los recientes desarrollos de la ética médica y empresarial, me llevan a pensar que no sea yo tan ingenuo cuando anuncio que la nueva sensibilidad empieza a moverse tras la virtud» (p. 212), dice este autor recordando un influyente libro de MacIntyre (1988). Se trata de una sensibilidad ética dentro de la cual el valor nuclear es la «emergencia», es decir, la acentuación de la primacía, tanto de la personalización, la libertad creativa y la responsabilidad personal, como de los bienes compartibles sobre los estrictamente «excluyentes»; esto es, el primado de los bienes que no decrecen al compartirlos y repartirlos, la consecución de metas comunes que unifican lo diverso respetando sin embargo la pluralidad.

A esta clase de bienes se han referido ya diversos autores. Y así, mientras R. Bernstein (1983) señala que «en una época en que la amenaza de una aniquilación total no parece una abstracta probabilidad, sino una posibilidad real inmediata, se hace cada vez más necesario tratar, una y otra vez, de alentar y nutrir aquellas formas de vida comunitaria en las que el diálogo, la conversación, la phrónesis, el discurso práctico y el juicio formen parte de nuestras prácticas cotidianas» (p. 229), MacIntyre (1988), por su parte, cerraba su libro «After Virtue» afirmando que «lo que importa ahora es la construcción de formas locales de comunidad, dentro de las cuales la civilidad, la vida moral y la vida intelectual puedan sostenerse a través de las nuevas edades oscuras que caen ya sobre nosotros» (p. 322).

Desde el punto de vista estrictamente pedagógico, todo lo que llevamos visto debe tener sus concretas implicaciones prácticas, así como teóricas. Subrayaré las siguientes:

1. En primer lugar, me parece urgente destacar con prioridad la necesidad de que, mediante la acción educativa, se aprenda a atribuir, de forma significativa y crítica, un valor intrínseco a las instituciones sociales democráticas comúnmente compartidas, formadas por los valores de la libertad, la igualdad de oportunidades vitales para todos, la racionalidad comunicativa y la responsabilidad solidaria. La conexión de la educación cívica — la ciudadanía madura - con el ejercicio de las virtudes implica afirmar el valor que éstas tienen como disposiciones y buenos rasgos del carácter, ejemplares por y para una comunidad de personas. Pero éstas se encuentran a su vez relacionadas con los valores -que son bienes-, de modo que la discusión sobre el tipo de virtudes cívicas que deben conformar el carácter del ciudadano maduro es inseparable de la discusión sobre los valores con los que dichas virtudes se relacionan. Y es claro que la tarea de la unificación europea -dentro de la cual la civilidad es una noción central- exige afirmar la existencia de bienes y valores que todos compartimos, bienes y valores de tal valor intrínseco formativo para la persona - que los podemos llegar a considerar como condiciones previas del bienestar: «Los bienes públicamente compartidos son 'ideales', esto es, como máximo, aseguran las condiciones socio-políticas para el bienestar de todos, y no todas las condiciones de ese bienestar», ha señalado Heller (1989, p. 222). 
2. En segundo término, la acción educativa debe principalmente, aunque no solamente, hacer de la educación del juicio la sustancia de la educación cívica. La razón principal de ello está en que, concebida como forma de vida, la democracia es sobre todo un encuentro entre personas que poseen diferentes intereses, opiniones y enfoque sobre las cosas. En ella la pluralidad —el pluralismo- no es sólo la afirmación de una realidad, la constatación de un hecho, sino una aspiración; por eso se habla de la «tolerancia» como virtud pública central en la democracia pluralista. En este encuentro, los ciudadanos revisan de continuo tales opiniones e intereses, aunque desde luego sobre la base de algo que ha de serles común, como por ejemplo los «valores superiores» que sostienen moralmente a un Estado de Derecho. Esta revisión —que exige diálogo y deliberación — se hace siempre en el contexto de un conocimiento no acabado, en cierto modo imperfecto, esto es, sobre la base de un saber que necesariamente ha de contar con ciertas zonas de indeterminación e incertidumbre. El principio de incertidumbre rige toda práctica que en esencia sea reflexiva y moral. Todo esto exige educación del juicio, es decir, capacidad de consejo práctico, habilidad para pasar de la deliberación a la decisión y de la teoría a la práctica. Si la discrepancia es una premisa esencial de la política, y la ciudadanía es la clave de la resolución de los conflictos sobre los valores y los fines, entonces la educación del juicio cívico y político es realmente crucial. De hecho, la participación tiene como función principal la educación del juicio. El ciudadano es, así, el individuo que ha aprendido ha elaborar juicios cívicos y quien puede evaluar los bienes en términos públicos. Del mismo modo que destacamos al estadista por su capacidad de discernimiento y juicio - por su prudencia política- hemos de destacar igualmente al ciudadano por su capacidad de juicio cívico, por su prudencia cívica —el buen juicio en la acción que aprendemos con la práctica (R. Beiner, 1983, pp. 72 y ss y 129-152) - y que es la que le habilitará para juzgar opciones y posibilidades. Como ha señalado Barber (1988) en «The conquest of politics», «el ciudadano es un hábil partícipe en la política, formado en las artes de la interacción social y marcado por la capacidad para distinguir los requerimientos de «nuestros» estilos de pensamiento de los que son «mis» estilos de pensamiento. Hablar del juicio político democrático es hablar de la educación cívica y también de estilos de participación política que van más allá del voto ocasional. Experto en juicio político es del que hablamos cuando nos referimos al ciudadano competente» (pp. 210-212): alguien comprometido con el ámbito público y gradualmente adiestrado por la experiencia política, aquélla que consiste en una prolongación de la ética a la vida social.

3. Muy específicamente, considero que la acción educativa sobre la conformación del carácter cívico debería atender a tres grandes objetivos: a) desarrollo de una perspectiva histórica, que supone el conocimiento de la propia tradición cultural y de las contribuciones realizadas por las diversas sociedades a lo largo del tiempo (Conseil de L'Europe, 1990; b) desarrollo de habilidades de acción social, en orden a producir personas ilustradas, comunicativas, cooperativas, responsables y solidarias (R. Pratte, 1988, pp. 9-24; R. F. Butts, 1980, pp. 121-152, 1988 y 1989); c) incremento de un sentido de pertenencia que asegure las propias raíces, pero evitando simultáneamente los riesgos del «etrocentrismo» y de ese «espíritu particularista» 
al que Ortega se refirió cuando hablaba de su proyecto de los Estados Unidos de Europa (J. Ortega y Gasset, 1949 y H. C. Raley, 1977 p. 182).

De acuerdo con estas ideas, considero esencial que nos preguntemos en qué ha de consistir finalmente esa nueva Europa y su específica promesa; ¿Qué esperamos conseguir?

Agnes Heller (1989) ha intentado responder a este interrogante planteándonos un interesante reto: «Lo que promete un nuevo marco europeo es el surgimiento de la virtud cívica, el gusto, la educación de los sentidos, la urbanidad, la civilidad, la alegría, la nobleza, unas formas de vida nacidas con dignidad, sensibilidad por la naturaleza, creada por el hombre o conservada» (p. 229). Es en este sentido y medida la razón por la cual, en cuanto «práctica de compromiso», esta promesa constituye una quehacer compartido que, lejos de emprenderse sólo como medio para un fin, ha de consistir en una actividad éticamente buena por sí misma. El civismo se desarrolla sanamente siempre -o por el contrario se ve frustradoen el seno de una comunidad que ha de aspirar a conservar de forma crítica su propia memoria y tradiciones. Para quienes viven fuera de su propio país y cultura, este hecho es crucial. Así pues, la vida social entera ha de consistir en una verdadera práctica de compromiso capaz de reconocer el modelo de interpretaciones y juicios que ha venido elaborando generación tras generación. Y es importante que, como ciudadanos educados y maduros, sepamos invitar creativamente al diálogo con la propia tradición y cultura a quienes, por necesidad, habrán de hacer sus vidas en ellas desde la que ya poseen y traen consigo. Porque la tradición, lejos de oponerse a racionalidad, la sugiere. «A menudo es — señala al respecto Bellah (1989) — un debate continuo y razonado sobre el bien de la comunidad o institución cuya identidad define» (p. 396)

\section{BIBLIOGRAFÍA}

AA. VV. (1990): Comunidad y utopía. Ensayos históricos, éticos y políticos (Universidad de la Laguna, Lerna).

ARENDT, H. (1988): Sobre la revolución (Madrid, Alianza).

- (1984): Thinking and moral considerations. A lecture. Social Research 51:1, pp. 7-37.

- (1974): La condición humana (Barcelona, Seix Barral).

BARBER, B. (1989): Public talk and civic action. Education for participation in a strong democracy. Social Education 53:6, pp. 355-356.

- (1988): The conquest of politics. Liberal philosophy in democratic times (Nueva Jersey, Princeton University Press).

- (1984): Strong democracy. Participatory politics for a new age (Berkeley, University of California press).

BEINER, R. (1983): Political judgment (Londres, Methuen).

BELLAH, R. y otros (1989): Hábitos del corazón (Madrid, Alianza).

4. Sobre el carácter narrativo de las comunidades y el sentido de la tradición en las prácticas sociales, en especial referencia la práctica educativa, consúltese: S. HAVERwAS, 1986, pp. 111-128 y G. LANGFORD, 1989, pp. 26-32. 
BELLO REGUERA, G. (1990): La idea de comunidad. Resistencia, historia y despliegue pragmático, en AA. VV. Comunidad y utopía. Ensayos históricos, éticos y políticos pp. 11-76 (Universidad de la Laguna, Lerna).

BENHABIB, S. (1988): Jugment and the moral foundations of politics in Arendt's thought. Political Theory 16:1, pp. 29-51.

BERNSTEIN, R. (1983): Beyond objetivism and relativism (Filadelfia, University of Pennsylvania Press).

BERRY, Ch. (1989): The idea of a democratic community (Harvester Wheatsheaf, St. Martin's Press).

BOLI, L. (1989): New citizen for a new society (Oxford, Pergamon Press).

BOLIVAR BOTIA, A. (1990): La educación ética y cívica en los países europeos. Cuadernos de Pedagogía no 186 , noviembre, pp. 8-13.

BUTTS, R. F. (1989): The civic mission in educational reform. Perspectives for the public and the profession (Neva York, Hoover Institute press).

- (1988): The morality of democratic citizenship. Goals for civic education in the republic's third century (California, Center for Civic Education).

- (1980): The revival or civic learning (Columbia, Teachers College Columbia University).

CAMPS, V. (1990): Virtudes públicas (Madrid, Espasa).

CAPUTO, G. (1988): Pour une Magna Charta des universités européennes. Création $\mathrm{n}^{\circ}$ 82, pp. 75-86.

CONSEIL DE L'EUROPE (1990): Séminaire européen d' enseignants sur «L'Europe: connaitre le passé, comprendre le present. Quelles connaissances avoir sus l' histoire de L'Europe en fin de scolarité obligatoire» (Strasbourg, Conseil de la Coopération Culturelle).

COOPER, J. W. (1989): Etica profesional y filosofía pública. Cuadernos de la Fundación Encuentro $\mathrm{n}^{\mathrm{o}} 63$, junio, pp. 45-57.

CORTINA, A. (1990): Democracia como forma de vida, en Etica sin moral pp. 254-273 (Madrid, Tecnos).

- (1990) El ethos democrático: télos, valor, virtud, en Etica sin moral. pp. 219-239 (Madrid, Tecnos).

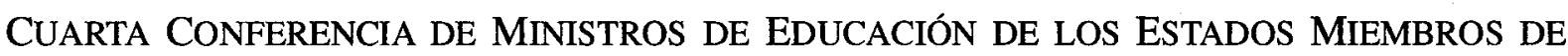
LA REGIÓN EUROPEA (1988): Perspectivas y tareas del desarrollo de la educación en Europa en los albores de un nuevo milenio. Cuadernos de la Fundación Encuentro, n⿳⼈ 43, noviembre, pp. 1-72.

D'ENTREVES, J. M. (1989): Agency, identity and culture. Hannah Arendt's conception or citizenship. u, 9:1/2, pp. 1-24.

DEWEY, J. (1980): Democracia y educación (Buenos Aires, Losada).

- (1954): The public and its problems (Chicago, Swallow Press).

DWORKIN, R. (1977): Taking Rights Seriously (Londes, Duckworth).

FLORES DÁRCAIS, P.: La democracia tomada en serio. Claves de Razón Práctica, n 2, mayo, pp. 2-14.

GADAMER, H. G. (1990): La herencia europea (Barcelona, Península).

GRUPO SOCIALISTA EUROPEO (1989): Manifiesto 89. Cuadernos de la Fundación Encuentro, $\mathrm{n}^{\circ}$ 56, abril, pp. 17-39.

GuTMan, A. (1987): Democratic education (Nueva Jersey, Princeton University Press).

HAVERWAS, S. (1986): A community of character (Londes, University of Notre Dame Press).

HEATER, D. (1990): Citizenship. The civil ideal in world history politics and education (Nueva York, Longman). 
Heller, A. (1989): Etica ciudadana y virtudes cívicas, en HELlER, A, y FEHER, F. (eds.): Políticas de la postmodernidad pp. 215-231 (Barcelona, Península).

HERNÁNDEZ PEDRERO, V. (1990): La comunidad ética. Entre la tradición y el consenso (El papel de la phrónesis), en AA. VV. ob. cit. pp. 77-110.

JANOWITZ, M. (1983): The reconstruction of patriotism. Education for civic consciousness (Chicago, University of Chicago Press).

LANGFORD, G. (1989): Teaching and the Idea of a Social Practice, en CARR, W. (ed.): Quality in Teaching. Arguments for a reflective profession, pp. 21-35 (Londres, The Falmer Press).

LlANO, A. (1988): La nueva sensibilidad (Madrid, Espasa).

MACINTYRE, A. (1990 a): Después de Tras la virtud. Entrevista con Alasdair MacIntyre, Atlántida 1:4, pp. 87-95.

- (1990 b): Three reval versions of moral enquiry (Nueva York, Notre Dame Press).

- (1988): Tras la virtud (Barcelona, Crítica).

NATIONAL TASK FORCE ON CITIZENSHIP EDUCATION (1977): Education for Responsible Citizenship (Nueva York, MacGraw-Hill).

OAKESHOTT, M. (1962): Political education, en Rationalism in politics, pp. 111-136 (Londes, Methuen).

ORTEGA Y GASSET, J. (1949): De Europa meditatio quaedam, en Europa y la idea de Nación pp. 29-120 (Madrid, Alianza-Revista de Occidente, 1985).

PARTIDO POPULAR EUROPEO (1989): Al lado del ciudadano. Cuadernos de la Fundación Encuentro, nำ 56, abril, pp. 43-86.

PRATTE, R. (1988): The civic imperative. Examining the need for civic education (Nueva York, Teachers College Press).

RALEY, H. C. (1977): Más allá del nacionalismo, hacia los Estados Unidos de Europa, en Ortega y Gasset, filósofo de la unidad europea, pp. 179-198 (Madrid, Revista de Occidente).

RAWLS, J. (1990): Sobre las libertades (Barcelona, Paidós).

RYN, C. G. (1990): Democracy and the ethical life. A philosophy of politics and community (Washington, D. C., The Catholic University of American Press).

SANDEL, M. (1982): Liberalism and the limits of justice (Nueva York, Cambridge University Press).

SARTORI, G. (1987): Teoría de la democracia 2 vols. (Madrid, Alianza).

SULLIVAN, W. M. (1986): Reconstructing public philosophy (Berkeley, University of California Press).

WHEELER, D. K. (1976): El desarrollo del currículum escolar pp. 93-106 (Madrid, Santillana). 